\title{
Fractura - hundimiento craneal congénita: una realidad obstétrica
}

\author{
Pablo Padilla I. ${ }^{1}$, Blanca Ferri F. ${ }^{1}$, Inmaculada Morcillo L. ${ }^{1}$, Antonio Abad C.1, \\ Vicente Payá A. ${ }^{1}$ \\ 1 Servicio de Obstetricia y Ginecología, Hospital Universitario y Politécnico La Fe. Valencia, España.
}

\section{RESUMEN}

Las fracturas craneales congénitas tienen baja incidencia, de una etiología desconocida, generando una gran alarma respecto a la actuación obstétrica en el momento del parto. Se presenta el diagnóstico, exploración radiológica y evolución clínica de dos recién nacidos con fractura-hundimiento craneal intrauterina, uno de ellos con manejo expectante y el otro con manejo quirúrgico. Ambos con buena evolución posterior y sin secuelas neurológicas ni estéticas.

\section{PALABRAS CLAVE: Fractura craneal congénita, fractura hundimiento craneal, defecto congénito, actuación obstétrica}

\section{SUMMARY}

The congenital skull fractures presented a low incidence, unknown aetiology, and it causes great alarm as far as the obstetric actions to be taken at birth are concerned. This work presents the diagnosis, radiology examinations and clinical evolution of two live-born infants with an intrauterine depressed skull fracture, one with expectant management and the other with surgical management. Both neonates showed good subsequent evolution with no neurological and no aesthetic sequelae.

\section{KEY WORDS: Congenital skull fracture, depressed skull fracture, congenital defect, obstetric attention}

\section{INTRODUCCIÓN}

Las fracturas por hundimiento craneal congénitas, conocidas también como fracturas en pelota de ping-pong, presentan una baja incidencia, estimada de 0,5-2 por cada 10.000 nacidos vivos (1). Siendo de etiología desconocida en la mayoría de las ocasiones (2).

Su diagnóstico suele ser mediante la exploración física en el momento del nacimiento, generando una gran alarma por la hipotética mala atención obstétrica del parto, con las consiguientes responsabilidades médico-legales, a pesar, que no a podido relacionarse su aparición con trauma obstétrico (3).

Presentamos el diagnóstico de dos neonatos con fractura-hundimiento del parietal derecho intrauterino, descripción radiológica y manejo, así como evolución clínica posterior. 


\section{Caso 1}

Secundigesta, primípara de 34 años, control gestacional dentro de la normalidad, sin antecedentes traumáticos. Consulta en la semana 40 por rotura prematura de membranas meconial; se inicia inducción del parto. Tras 2 horas, realiza alteraciones de la frecuencia cardíaca fetal (Dips III), y ante la imposibilidad de realizar $\mathrm{pH}$ de cuero cabelludo fetal por partes blandas desfavorables, se indica cesárea ante riesgo de pérdida de bienestar fetal. Se extrae recién nacido (RN) femenino de 3.150 gramos (p50-75), Apgar 9/10, con pHa 7.21. Extracción fetal expedita en 5 minutos y sin incidentes. La RN presentaba en la exploración depresión del parietal derecho de aproximadamente $10 \mathrm{~mm}$ de profundidad, sin crepitación ni signos de hematoma o sangrado activo en cuero cabelludo, con un perímetro cefálico de $34 \mathrm{~mm}$ (p50-75). Sin otras malformaciones externas. Exploración neurológica normal. Se practicó estudio radiológico donde se informa de fractura por hundimiento en parietal derecho con un trayecto lineal de $40 \mathrm{~mm}$ de longitud (Figura 1). Ecografía cerebral no se evidencia lesiones en SNC. Permanece asintomática, por lo que se decide actitud expectante con controles ambulatorios, dándose el alta a los 7 días. A los 4 meses de vida, se verifica resolución espontánea de la fractura, comprobándose consolidación radiológica. La evolución al año de vida no presenta lesiones neurológicas o estéticas, con desarrollo psicomotor adecuado para su edad.

\section{Caso 2}

Primigesta de 27 años, control gestacional dentro de la normalidad. Acude en la semana 39 por sensación de dinámica regular, a la exploración

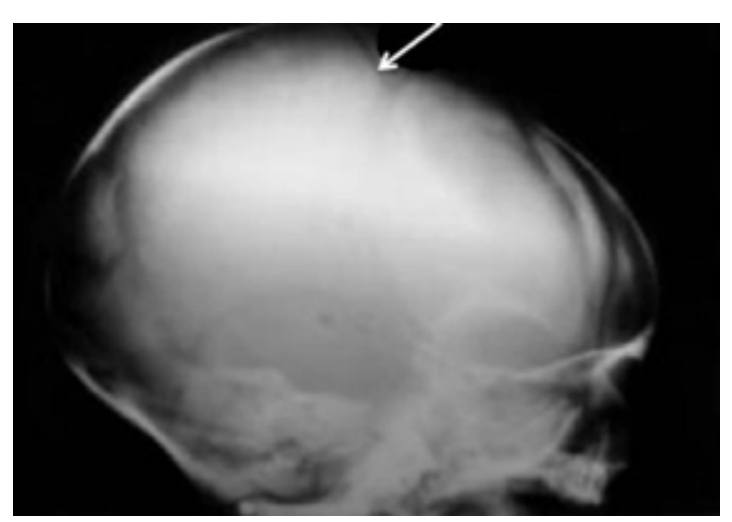

Figura 1. Caso 1: Radiografía sagital de cráneo realizada a las 4 horas de vida. Se observa línea de fractura sobre parietal derecho (flecha). cérvix borrado y dilatado $3 \mathrm{~cm}$, cefálica occipito ilíaca izquierda transversa. Durante la dilatación, realiza alteraciones de la frecuencia cardíaca fetal (Dips tipo II), por lo que se indica control mediante $\mathrm{pH}$ de cuero cabelludo fetal, encontrándose con una dilatación de $5 \mathrm{~cm}$. Su resultado fue de 7.12 , se decide cesárea urgente ante el riesgo de pérdida de bienestar fetal. Se extrae RN femenino de 2.825 gramos (p25-50), Apgar 9/10, pHa 7.14 y EB $-16,8$. Extracción fetal en 3 minutos, expedita y sin incidentes. En la exploración presentaba depresión del parietal derecho de aproximadamente $30 \mathrm{~mm}$ de profundidad, sin crepitación, hematoma o ede$\mathrm{ma}$, con fontanela anterior normotensa (Figura 2). Perímetro cefálico de 31,5 mm (p25-50). Sin otras malformaciones externas. Exploración neurológica normal. El estudio radiológico informa de doble trazado de fractura lineal por hundimiento en parietal derecho con un trayecto $35 \mathrm{~mm}$ de longitud. Ecografía cerebral no lesiones intracraneales. Se efectúa corrección quirúrgica precoz a las 8 horas de vida. Tras incisión cutánea en parietal derecho se procede a trepanación, tras ello, elevación del reborde óseo con disector, corrigiendo el hundimiento. Permanece asintomática, con TAC craneal posterior a la cirugía sin evidenciar complicaciones. Alta al 4 ㅇ día con controles ambulatorios (Figura 3). Evaluación neurológica normal a los dos meses de vida.

\section{DISCUSIÓN}

El diagnóstico en el nacimiento de fracturas con hundimiento de la bóveda craneal son muy infrecuentes, con una incidencia estimada en nuestro medio de 0,56 por cada 10.000 nacidos vivos. Siendo la afectación más frecuente el hueso parietal (1).

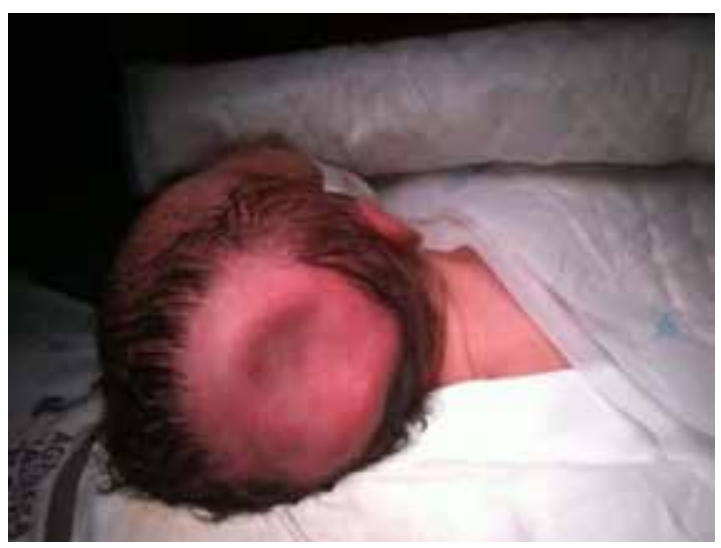

Figura 2. Caso 2: Se evidencia hundimiento parietal derecho de aproximadamente $3,5 \mathrm{~cm}$. 


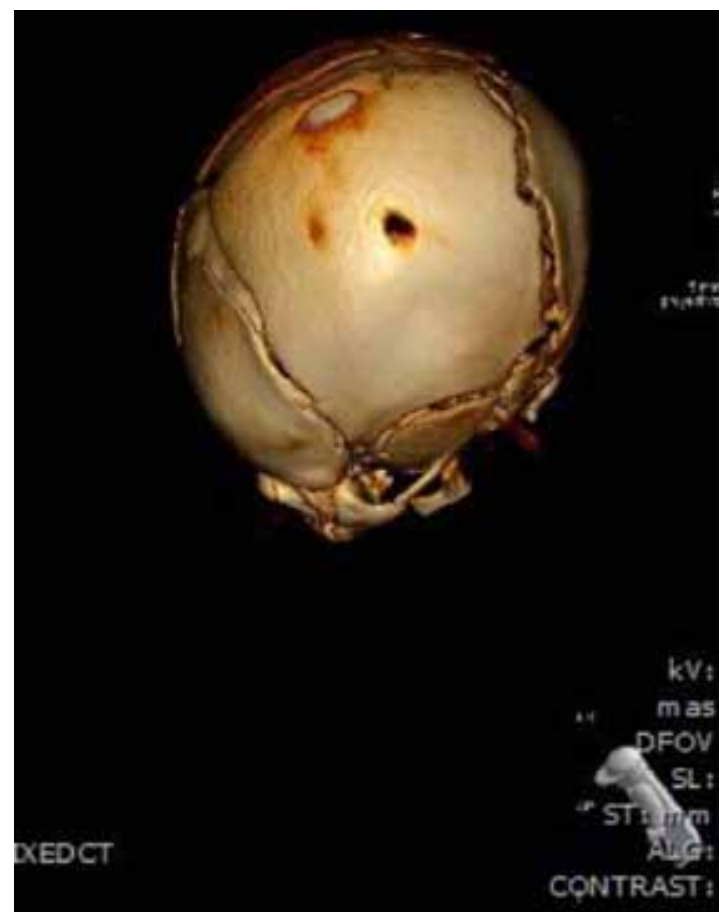

Figura 3. Caso 2: Reconstrucción 3D TAC craneal previo al alta, realizado 3 días después de la intervención. Véase la normalidad de la bóveda craneal.

Respecto a su patogenia, existen casos descritos de aparición tras caída accidental de la gestante o traumatismo directo sobre el útero grávido $(2,4)$. Actualmente la mayor parte de los casos publicados, no presentan antecedentes de este tipo ni una incorrecta asistencia obstétrica en el momento del parto.

La teoría más aceptada se centra en la aparición de focos de presión intrauterina prolongados sobre la bóveda fetal en rápido crecimiento, como génesis de este tipo de fracturas. Generalmente alteraciones en el esqueleto pélvico materno, como la compresión de la cabeza fetal sobre el promontorio-sacro materno, la presencia de los propios miembros fetales (gestaciones gemelares) o alteraciones uterinas (miomas intramurales de gran tamaño) (1-3).

El hecho indispensable para su génesis es una compresión prolongada sobre una zona del cráneo fetal (3), respondiendo a un mecanismo inelástico, con deformación de la cabeza en "ping-pong", al ejercer una fuerza externa constante sobre la calota fetal, se genera una depresión/incurvación ósea, llegando a la fractura si persiste el daño, por lo que el abanico de lesiones abarca desde una indentación en la bóveda craneal hasta la fractura con hundimiento (5).

Clásicamente este tipo de fracturas, se vinculaban con lesiones hipotéticas sobre parénquima cerebral subyacente, con atrofia cortical y adhesiones entre cerebro-meninges, con mayores tasas de epilepsia sobre todo si el hundimiento era mayor de $5 \mathrm{~mm}$. Estos datos no se han podido confirmar, dado que cursan de forma asintomática, sin lesión del parénquima cerebral subyacente (5). Respecto al manejo existe controversia. Se puede adoptar por una actitud expectante buscando su resolución espontánea o la corrección quirúrgica precoz.

Existen autores que defienden la reducción quirúrgica inmediata, dada la sencillez de la técnica y su baja tasa de complicaciones. Nadas y Reimberg (1), recomiendan la reparación quirúrgica, aun en ausencia de síntomas, cuando la depresión ósea sea mayor de $20 \mathrm{~mm}$. Otros centran la indicación si existen fragmentos óseos en el parénquima cerebral, lesión neurológica, aumento de la presión intracraneal o evidencia de LCR subgaleal (6). Otras medidas más drásticas, consideran que toda fractura-hundimiento congénita debe ser reparada de forma precoz dada la corrección inmediata de la alteración estética (7).

Por el contrario, se han publicado numerosos casos de resolución espontánea, con buena evolución en periodos relativamente cortos de tiempo. Hung y cols (8), observan con tratamiento conservador, restauración entre 1-6 meses para menores de $2 \mathrm{~mm}$ profundidad. También se describe resolución espontánea para fracturas con mayor profundidad (lesión de $40 \mathrm{~mm}$ con resolución en 4 meses) (9).

\section{CONCLUSIÓN}

No existe un criterio aceptado sobre el manejo. Nuestra experiencia muestra, que tanto el manejo expectante como el quirúrgico precoz, fueron igualmente correctos. La ecografía cerebral es muy importante para el seguimiento de las posibles complicaciones derivadas del manejo expectante o del acto quirúrgico. Debemos evitar las posibles repercusiones legales que conlleva, la hipotética relación de este tipo de fractura con una mala atención en el momento del parto, explicando a la familia clara y verazmente lo ocurrido.

\section{REFERENCIAS}

1. Nadas S, Reimberg O. Obstetrics fractures. J Pediatr Surg 1992;2:165-8.

2. Abbassioum K, Amirjamshidi A, Raimizadeh A. Spontaneus intrauterine depressed skull fractures. Child's Nerv Syst 1986;2:153-6.

3. Aliabadi H, Miller J, Radnakrishnan S, Mehta Al, Thomas K, Selznick L, Goldberg R, Grant G. Spontaneous intrauterine "ping-pong" fracture: review and case illustration. Neuropediatrics 2009;40:73-5. 
4. Moss SD, Walker ML, Ostergard S, Golembeski D. Intrauterine growing skull fracture. Child's Nerv Syst 1990;6:468-70.

5. Agrawal SK, Kumar P, Sundaram V. Congenital depression of the skull in neonate: a case of successful conservative management. J Child Neurol 2010;25:387-9.

6. Loeser JD, Kilburn HL, Jollet T. Management of depressed skull fractures in the newborn. J Neurosurg 1976;44:62-4.

7. Choux M. Incidence, diagnosis and management of skull fractures. En: Choux M. Head injuries in the newborn and infant. New York: Springer Verlag, 1985. pp 163-82.

8. Hung KL, Liao HT, Huang JS. Rational management of simple depressed skull fractures in infants. J Neurosurg 2005;103:69-72.

9. Hanlon L, Hogan B, Corcoran D, Ryan S. Congenital depression of the neonatal skull: a self limiting condition. Arch Dis Child Fetal Neonatal Ed 2006;91(4):F272. 